\title{
Preparation of Reactive Monodisperse Particles in the Micron Range by Dispersion Polymerization of Glycidyl Methacrylate
}

\author{
Kazuhiko TaKahashi, ${ }^{*, * *, \dagger}$ Hiroshi Uyama, ${ }^{* * *}$ and Shiro KobayashI ${ }^{* * * . \dagger}$ \\ * Department of Materials Chemistry, Graduate School of Engineering, \\ Tohoku University, Sendai 980-8579, Japan \\ ** Fujikura Kasei Co., Ltd., 13-1 Sakurada 5-chome, Washinomiya-machi, \\ Kitakatsushika-gun, Saitama, 340-0203, Japan \\ *** Department of Materials Chemistry, Graduate School of Engineering, \\ Kyoto University, Kyoto 606-8501, Japan
}

(Received January 26, 1998)

\begin{abstract}
KEY WORDS Dispersion Polymerization / Reactive Particle / Monodisperse Particle / Glycidyl
Methacrylate
\end{abstract}

Dispersion polymerizations have received much attention as a simple and convenient method to prepare monodisperse polymer particles from styrene and methyl methacrylate in the micron range. Preparation of micron-size monodisperse polymer particles by dispersion polymerization in polar media has been extensively studied. ${ }^{1-8}$ However, as to from other vinyl monomers there have been only a few papers on the dispersion polymerization to prepare monodisperse particles. ${ }^{9-12}$ Design of the solvent composition of the polymerization is very important for achievement of monodispersity.

Monodisperse polymer particles having reactive groups on the surface have much potential for various applications, since functional moiety can be readily immobilized on the surface of such particles. ${ }^{9}$ Recently, we have accomplished in the preparation of reactive monodisperse polymer particles in the micron range by dispersion polymerizations of 2-hydroxyethyl methacrylate ${ }^{11}$ and 4-vinylpyridine. ${ }^{12}$

Glycidyl methacrylate (GMA) polymer is widely used as a prepolymer of functional polymers, since the polymer has a highly reactive glycidyl group. Monodisperse poly(glycidyl methacrylate) (PGMA) particles were prepared by precipitation polymerization in ethyl acetate, however, the particle yield was up to $50 \% .{ }^{13}$ This study deals with preparation of monodisperse reactive particles in the micron range by the dispersion polymerization of GMA. By utilizing dispersion polymerization technique, PGMA particles with narrow size distribution were quantitatively obtained.

\section{EXPERIMENTAL}

A typical run of particle preparation was as follows (entry 5). $20 \mathrm{~g}$ of GMA, $2.0 \mathrm{~g}$ of poly ( $N$-vinylpyrrolidone) (PVP), $0.40 \mathrm{~g}$ of $\alpha, \alpha^{\prime}$-azobis(isobutyronitrile) (AIBN), $72 \mathrm{~g}$ of methanol, and $8 \mathrm{~g}$ of $\mathrm{N}, \mathrm{N}$-dimethylformamide (DMF) were weighted in a $350 \mathrm{~mL}$ glass bottle and the mixture was deoxygenated by bubbling nitrogen. Then, the bottle was placed on the shelf which was shaken at 160 times per min in a water bath at $65^{\circ} \mathrm{C}$ for $8 \mathrm{~h}$. After

+ To whom all correspondence should be addressed. polymerization, the reaction mixture was poured into a large amount of methanol, followed by centrifugation of the dispersion. The particles were twice subjected to the resuspension in methanol and the successive centrifugation.

Scanning electron microscopic (SEM) analysis was carried out by a JEOL JSM-T330 apparatus. In each sample, size of $200-300$ particles was measured to calculate the size, size distribution, and coefficient of variation of particles. IR spectra were recorded on Horiba FT-720 spectrometer.

\section{RESULTS AND DISCUSSION}

At first, the polymerization was carried out using PVP by $\mathrm{AIBN}$ initiator at $65^{\circ} \mathrm{C}$ in a methanol-containing solvent. Polymerization results are summarized in Table I. As an index of dispersity, coefficient of variation (standard deviation/average, $C_{\mathrm{v}}$ ) and size distribution $\left(D_{w} / D_{n}\right)$ are given. The resulting particles were analyzed by SEM.

In case of the solo use of methanol, the polydisperse particles were obtained in a high yield (entry 1). For preparation of monodisperse polymer particles by dispersion polymerization in polar media, water was often used as cosolvent. In case of the dispersion polymerization of GMA in an aqueous methanol, 80\%-methanol solvent reduced the dispersity of the particles (entry 2), however, the coagulation took place in $60 \%$ methanol (entry 3).

Very recently, we have found that monodisperse poly(acrylonitrile) particles were obtained in a mixture of methanol and DMF, ${ }^{14}$ the solvent system of which was applied to the present dispersion polymerization. Figure 1(A) shows SEM photograph of PGMA particles obtained in a mixture of methanol and DMF $(90: 10$ $w t \%$ ). The resulting particles were monodisperse in the micron range, indicating that the addition of DMF produced monodisperse PGMA particles. Furthermore, particle size could be controlled by changing the monomer concentration (entries 4-6). Quantitative yield of the monodisperse PGMA particles was achieved in the monomer concentration of 20 or $30 \%$ (entries 5 and 
Table I. Dispersion polymerization of GMA

\begin{tabular}{|c|c|c|c|c|c|c|c|c|}
\hline \multirow{2}{*}{ Entry } & \multirow{2}{*}{$\begin{array}{l}\text { Reaction } \\
\text { conditions }^{\mathrm{a}}\end{array}$} & \multirow{2}{*}{ Solvent } & \multirow{2}{*}{$\begin{array}{c}\text { GMA } \\
\text { Concn }^{\mathrm{c}} \\
\%\end{array}$} & \multirow{2}{*}{$\frac{\text { Yield }}{\%}$} & \multirow{2}{*}{$\frac{D_{n}^{\mathrm{d}}}{\mu \mathrm{m}}$} & \multirow{2}{*}{$D_{w} / D_{n}^{\mathrm{d}}$} & \multirow{2}{*}{$\frac{C_{\mathrm{v}}^{\mathrm{d} \cdot \mathrm{e}}}{\%}$} & \multirow{2}{*}{$\begin{array}{c}\begin{array}{c}\text { Remaining epoxy } \\
\text { group }^{f}\end{array} \\
\%\end{array}$} \\
\hline & & & & & & & & \\
\hline 1 & I & Methanol & 20 & 98 & 1.88 & 1.144 & 21.3 & 86 \\
\hline 2 & I & Methanol/Water $(80)$ & 20 & 97 & 2.04 & 1.052 & 12.4 & 83 \\
\hline 3 & $\mathrm{I}$ & Methanol/Water (60) & 20 & Coagulated & & & & \\
\hline 4 & I & Methanol/DMF (90) & 10 & 90 & 1.70 & 1.009 & 7.4 & 9 \\
\hline 5 & I & Methanol/DMF (90) & 20 & 100 & 2.45 & 1.010 & 5.7 & 18 \\
\hline 6 & I & Methanol/DMF (90) & 30 & 100 & 4.32 & 1.024 & 10.9 & 23 \\
\hline 7 & I & Methanol/DMF (80) & 20 & 91 & ca. 5 & & & 12 \\
\hline 8 & II & Methanol & 20 & Coagulated & & & & \\
\hline 9 & II & Methanol/DMF (90) & 20 & Coagulated & & & & \\
\hline 10 & II & Methanol/DMF (80) & 10 & 78 & 2.68 & 1.022 & 7.9 & 74 \\
\hline 11 & II & Methanol/DMF (80) & 20 & 93 & 3.91 & 1.018 & 7.8 & 81 \\
\hline 12 & 'II & Methanol/DMF (80) & 30 & 98 & 8.94 & 1.222 & 29.5 & 81 \\
\hline
\end{tabular}

${ }^{\mathrm{a}}$ I, polymerization using PVP stabilizer ( $10 \mathrm{wt} \%$ based on GMA) by AIBN initiator ( $2 \mathrm{wt} \%$ based on GMA) at $65 \mathrm{C}$ for $8 \mathrm{~h}$; II, polymerization using PVP stabilizer (10 wt \% based on GMA) by AMDVN initiator ( $1 \mathrm{wt} \%$ based on GMA) at $40 \mathrm{C}$ for $5 \mathrm{~h}$. ${ }^{\mathrm{b}}$ In parenthesis, weight ratio of methanol in the mixed solvent. ${ }^{\mathrm{c}}$ Weight $\%$ based on total. ${ }^{\mathrm{d}}$ Determined by SEM. ${ }^{\mathrm{e}}$ Coefficient of variation of number-average size. ${ }^{\mathrm{f}}$ Determined by using $\mathrm{HCl}-1,4$-dioxane reagent according to the literature. ${ }^{15}$
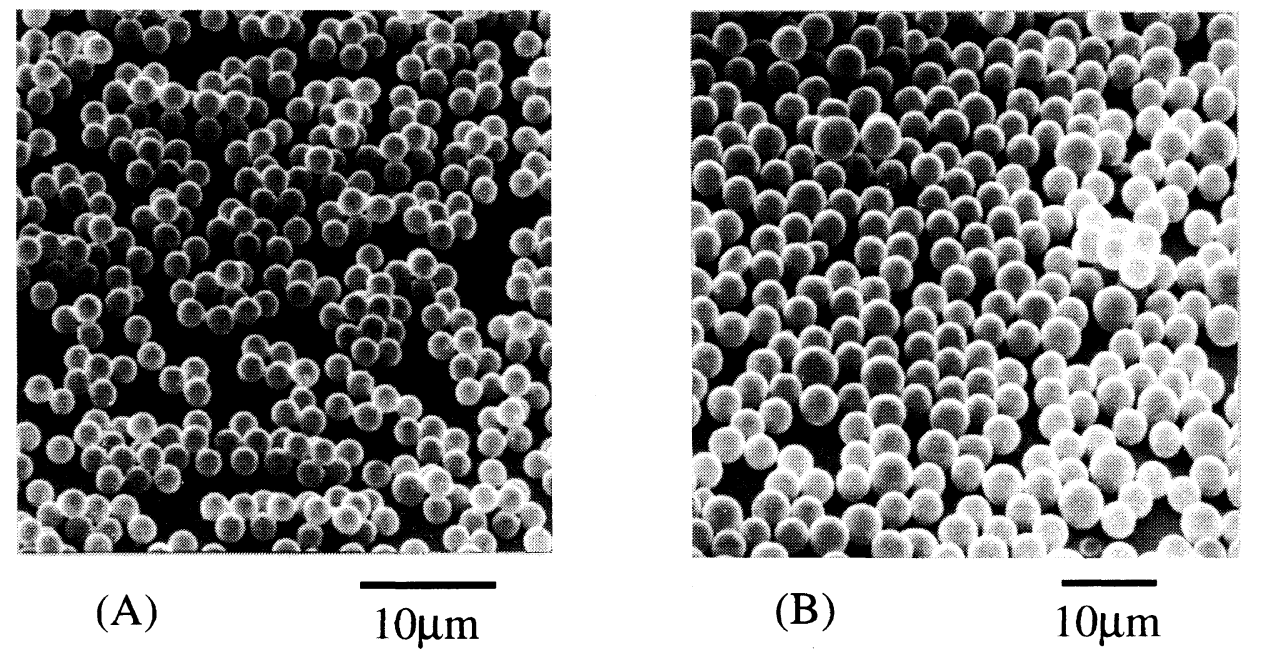

Figure 1. SEM photographs of PGMA particles: (A), entry 5; (B), entry 11.

6), whereas the polymer yield was not quantitative in $10 \%$ monomer concentration (entry 4$)$. This may be because the decrease of the apparent reaction rate in the lower monomer concentration resulted in the decrease of the particle yield.

In the solvent of the same methanol content, the particle diameter obtained in the aqueous methanol was smaller than that in a mixture of methanol and DMF (entries 2 and 7). In the dispersion polymerization, the particle size is supposed to be controlled by the critical degree of polymerization at which the oligomers form nuclei. ${ }^{1-8}$ The difference of the particle diameter is probably explained as follows; a mixed solvent of methanol and DMF showed larger solubility toward PGMA than an aqueous methanol. In using DMF as cosolvent, therefore, the larger critical degree of polymerization of PGMA resulted in the production of the larger PGMA particles.

In the emulsion polymerization of GMA, the oxirane ring was partly opened during the polymerization. The remaining ratio of the epoxide group in the resulting particles was determined. ${ }^{15}$ In case of the particles obtained in an aqueous methanol or methanol, the epoxide group remained in more than $80 \%$ (entries 2 and 3 ), on the other hand, the particles obtained in a mixture of methanol and DMF contained the low amount of the epoxide group (entries 4-6).

When the epoxide group is reacted, a hydroxy group is newly formed, which is readily detected by IR spectroscopy. Figure 2 shows FT-IR spectra of the PGMA particles. The ratio of broad peak centered at $3550 \mathrm{~cm}^{-1}$ due to $\mathrm{O}-\mathrm{H}$ vibration to that at $3000 \mathrm{~cm}^{-1}$ ascribed to aliphatic $\mathrm{C}-\mathrm{H}$ vibration depended on the polymerization condition; the ratio of the particles obtained in a mixture of methanol and DMF (Figure 2(B)) was larger than that in an aqueous methanol (Figure 2(A)). These data support that the epoxide group was subject to the ring-opening during the polymerization in the solvent containing DMF.

The particles obtained in methanol or an aqueous methanol were soluble or swollen in DMF, ethyl acetate, methyl ethyl ketone, pyridine, and toluene. In case of the particles obtained in a mixture of methanol and DMF, no appearance change was observed in the sample after immersion in such solvents, suggesting that the epoxide group was intermolecularly reacted with the 


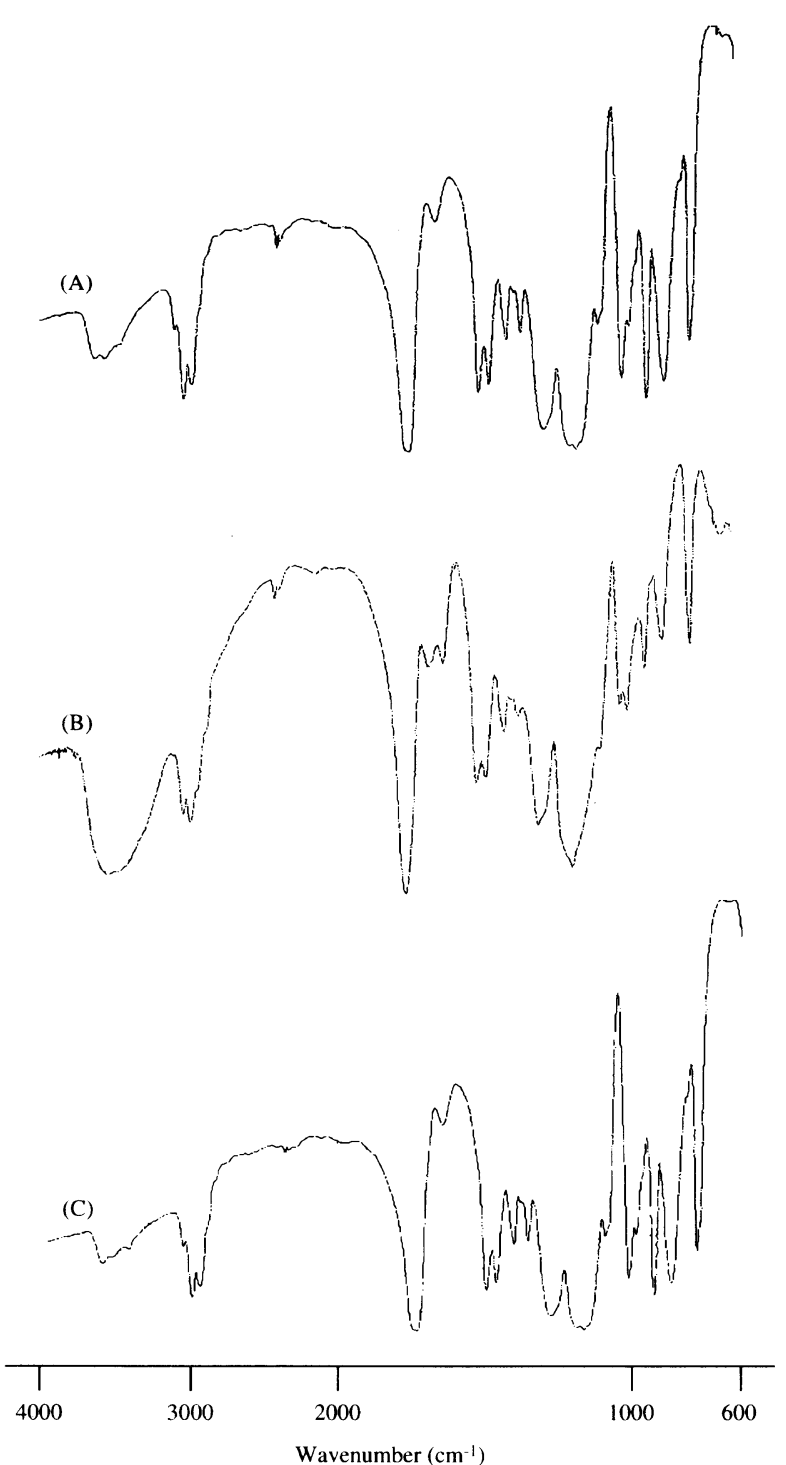

Figure 2. FT-IR spectra of PGMA particles: (A), entry 2; (B), entry 4; (C), entry 11 .

formed hydroxy group to give crosslinked polymer particles. $^{15}$

It is reported that the ring-opening reaction of glycidyl group was suppressed by decrease of the polymerization temperature in the emulsion polymerization of GMA. ${ }^{15}$
Then, the dispersion polymerization was carried out at lower temperature of $40^{\circ} \mathrm{C}$ by using $2,2^{\prime}$-azobis(4-methoxy-2,4-dimethylvaleronitrile) (AMDVN) as initiator (entries 8-12), which was decomposed at lower temperature than AIBN. In the polymerization in methanol or $10 \%$ DMF by AMDVN, a stable dispersion was not formed (entries 8 and 9). Monodisperse PGMA particles were obtained by using 10\% GMA (entry 10, Figure $1(B))$. The monomer concentration of $20 \%$ also afforded micron-size particles with narrow size distribution (entry 11). The dispersity became broader in the polymerization using 30\% GMA (entry 12). The sample of entry 10 or 11 was almost soluble in ethyl acetate. FT-IR analysis showed that the amount of the hydroxy group in the particles was low (Figure 2(C)). These data indicate that the polymerization at lower temperature produced monodisperse particles containing large amount of the reactive epoxide group.

\section{REFERENCES AND NOTES}

1. Y. Almog, S. Reich, and M. Levy, Br. Polym. J., 14, 131 (1982)

2. C. M. Tseng, Y. Y. Lu, M. S. El-Aasser, and J. W. Vanderhoff, J. Polym. Sci., Polym. Chem. Ed., 24, 2995 (1986).

3. A. J. Paine, W. Luymes, and J. McNulty, Macromolecules, 23, 3104 (1990).

4. A. Tuncel, R. Kahraman, and E. Piskin, J. Appl. Polym. Sci., 50, 303 (1993).

5. S. Kobayashi, H. Uyama, Y. Matsumoto, and I. Yamamoto, Makromol. Chem., 193, 2355 (1992).

6. H. Uyama and S. Kobayashi, Polym. Int., 34, 309 (1994).

7. K. P. Lok and C. K. Ober, Can. J. Chem., 63, 209 (1985).

8. S. Kobayashi, H. Uyama, J. H. Choi, and Y. Matsumoto, Polym. Int., 30, 265 (1993).

9. S. Margel, E. Nov, and I. Fisher, J. Polym. Sci., Polym. Chem. Ed., 29, 347 (1991).

10. S. Margel, M. Tennenbaum, and H. E. Gottlieb, J. Polym. Sci., Polym. Chem. Ed., 30, 1499 (1992).

11. K. Takahashi, S. Miyamori, H. Uyama, and S. Kobayashi, $J$. Polym. Sci., Polym. Chem. Ed., 34, 175 (1996).

12. K. Takahashi, S. Miyamori, H. Uyama, and S. Kobayashi, Macromol. Rapid Commun., 18, 471 (1997).

13. S. Hosaka, Y. Murao, S. Masuko, and K. Miura, Immunol. Commun., 12, 509 (1983).

14. K. Takahashi, H. Uyama, and S. Kobayashi, Polym. Prepr. Jpn., 45, 144 (1996).

15. T. Matsumoto, M. Okubo, and Y. Takahashi, Kobunshi Ronbunshu, 34, 571 (1977). 